\title{
EVALUATION OF MORPHOLOGICAL CHANGES OF THE PARANASAL SINUS IN COMPUTED TOMOGRAPHY AND MAGNETIC RESONANCE IMAGING MODALITIES
}

\author{
SANTOSH OJHA ${ }^{1 *}$, MANNA DEBNATH ${ }^{1}$, ANUPAM NIRAULA ${ }^{1}$, SHAHNAWAZ ALI KHAN TAHIRI ${ }^{2}$
}

${ }^{1}$ Department of Medical Imaging Technology, Charotar Institute of Paramedical Sciences, Charotar University of Science and Technology, Anand, Gujarat, India. ${ }^{2}$ Application Specialist, AltiusTech Health, Mumbai, Maharashtra, India. Email: santdastan@gmail.com

Received: 11 June 2020, Revised and Accepted: 06 July 2020

\section{ABSTRACT}

Objectives: The objective of the study was to evaluate the prevalence of pathological conditions and anatomical variations occurring within paranasal sinus among the patients undergoing computed tomography (CT) scan and magnetic resonance imaging (MRI) scan in Gurugram district.

Methods: A prospective study was conducted among 20 patients in each modality, the patient undergoing the CT of paranasal sinus and patients undergoing routine MRI brain study. The clinical findings from CT and MRI were taken as primary data and later on compiled together under different variables to assess the occurrence of paranasal sinus disease and anatomical variations within.

Results: The present study revealed that the most common pathology was mucosal thickening followed by polyp and the occurrence of air-fluid level; maxillary sinus being commonly affected. In patients undergoing CT, the most common anatomical variation was deviated nasal septum, that is, 16 out of 20 patients followed by the bone spur formation. In the patients undergoing routine MRI brain, most of the patient had mucosal thickening $<5$ $\mathrm{mm}$. In the lining of maxillary sinus, moderate hyperintensity was reported.

Conclusion: Based on the study, it is concluded that mucosal thickening is most commonly evident pathology with maximum occurrence seen in the maxillary sinus. The co-occurrence of more than 1 anatomical variation and involvement of multiple paranasal sinuses were seen positive. MRI was found more sensitive in assessing the early changes in sinus mucosal abnormalities.

Keywords: Paranasal sinus, Mucosal thickening, Deviated nasal septum, Sinusitis.

(C) 2020 The Authors. Published by Innovare Academic Sciences Pvt Ltd. This is an open access article under the CC BY license (http://creativecommons. org/licenses/by/4. 0/) DOI: http://dx.doi.org/10.22159/ajpcr.2020.v13i9.38723

\section{INTRODUCTION}

The paranasal sinuses (PNS) are the hollow space that are present around the nasal cavity. There are overall four pairs of paranasal sinus present in the skull and are titled according to the bone in which they are present, that is, maxillary, frontal, sphenoid, and ethmoid sinus [1]. The major functions of sinus include lightening the weight of the head, humidifying and heating inhaled air, increasing the resonance of speech, and serving as a crumple zone to protect vital structures in the event of facial trauma. The major integrant of paranasal sinus includes the mucus secretions, the hair-like structure that drains the mucus out of the paranasal sinus, and the sinus ostium. Any inflammation in above-mentioned components render the sinus liable to infections [2].

As the nose and the paranasal sinus comprise the common entry site to the human body, that is, respiratory system, the sinuses are more prone to infections. The anatomical changes occurring within these structures are also a major stimulant for infection as it hinders the normal functioning of the PNS. Another important structure that is highly afflicted or triggers the infection is the osteomeatal complex which comprises the ostium of most of the sinus giving a common drainage channel. Any obstruction to this complex disrupts the airflow through the sinus and the flow of mucosa within the cavities which makes the patient prone to sinus disease. The presence of pneumatization of any size within the middle turbinate bone is called concha bullosa. When the size of pneumatization gets sufficiently enough to block any of the passage, the concha bullosa becomes clinically important. Any significant deviation of nasal septum from the midline, due to morphological changes within the cavities, is septal deviation [3,4].

Due to the intricacy of structures within paranasal sinus, radiologic findings have always been the most promising for finding the diseased condition with its extent and anatomical variations. Computed tomography (CT) produces excellent bone detail and accurate soft-tissue mapping and is considered to provide the most accurate details of the extent of disease and that of anatomical conditions, thus providing basic landmarks for performing the surgery or further treatment [5]. The CT scan can delineate the changes occurring within PNS during the fungal and nonfungal sinusitis, thus giving the profile of the sinusitis [6]. On the other hand, because of the inherent soft-tissue contrast of magnetic resonance imaging (MRI), the secretions, thickened mucosa, and masses are better differentiated. The sensitivity of MRI lies in the better assessment in the extension of infections beyond the paranasal sinus to the surrounding structures [7]. Since the pollution is increasing at an alarming rate due to rapid urbanization, the impact of air pollution in respiratory functions is very evident in Delhi National Capital Region - Gurugram district [8]. The paranasal sinus is more prone to infection being a common entry site for inhaled air. This study is conducted to assess the prevalence and extent of the morphological changes in paranasal sinus among the Gurugram population under CT and MRI modalities.

\section{METHODS}

A prospective, observational study was conducted from November 2018 to April 2019 among the patients undergoing the non-contrast computed tomography (NCCT) scan of PNS and routine MRI brain study. The informed consent was taken from each patient before the commencement of the study.

\section{Inclusion criteria}

The patients undergoing the CT of PNS with symptoms such as headache, nasal obstruction, nasal discharge, anosmia, and epistaxis were included in the study. As for MRI, patients undergoing routine 
brain study with incidental positive findings of the PNS were included in the study.

\section{Exclusion criteria}

Any patients with a history of facial trauma, sinonasal surgery, uncooperative and psychiatric patients, pediatric $(<12$ years $)$, and geriatric patients ( $>65$ years) were excluded from the study.

The CT images were acquired on Siemens Somatom scope 16 slice multidetector spiral CT scanner. The NCCT images of paranasal sinus were acquired on helical mode with the following parameter; tube voltage 130 kilovoltage $(\mathrm{kV})$, tube current 60 milliampere-seconds (mAs), slice acquisition $3.0 \mathrm{~mm}$, and pitch 0.80 . The acquired volume data were later on reconstructed on axial, coronal, and sagittal images into two basic algorithms each, that is, bone algorithm and soft-tissue algorithms.

The routine MRI brain scan was carried out on a Philips Multiva 1.5 Tesla MRI scanner. The T2-weighted axial and coronal sequences were used for evaluation for changes in the paranasal sinus.

\section{Image assessment}

The acquired images were evaluated by senior radiologist and results were recorded on predesigned pro forma for both CT and MRI findings. The abnormal sinus findings were recorded for both CT and MRI are mucosal thickening, polyp, and fluid level, their distribution and pattern along with anatomical variations such as deviated nasal septum (DNS) and concha bullosa. For CT, bone spur formation was noted and for MRI, the mucosal thickening and its appearance in T2-weighted images were recorded along with the inferior turbinate hypertrophy. The mucosal thickening was measured using digital imaging and communications in medicine viewer in millimeters and expressed into three different categories - $>5 \mathrm{~mm}, 5-10 \mathrm{~mm}$, and $<10 \mathrm{~mm}$. The maximum measurement was recorded from the point of maximum thickness floor of the maxillary sinus. Similarly, the assessment of the hyperintensity of the mucosal coating of the maxillary sinus was done by the reporting radiologist. The assessment was based on the visual observation of the radiologist on T2-weighted axial and coronal images and was given a separate case pro forma to assign and categorize as mild, moderate, and severe.

\section{Statistical analysis}

The observations were recorded on a pre-designed pro forma. The descriptive analysis was conducted using the Microsoft Excel 2016 where frequency, mean, and standard deviation were calculated. The data were expressed in frequency and percentage in the form of table and figure.

\section{RESULTS}

In the present study, 40 patients were included in which 23 were male and 17 were female with a mean age of $38.0 \pm 14.6$ years. As for the age distribution, 30-40 years of age group patients were common age group to have sinus pathology followed by 21-30 years.

The radiological findings of paranasal sinus showed that the increased mucosal thickening was the most prevalent sinus abnormality which was followed by polyp/cyst and fluid level (Fig. 1). More than 1 pathology was noted among nine patients.
The maxillary sinus was the most commonly affected sinus followed by ethmoid, frontal, and sphenoid sinus (Table 1). Multiple involvements of the sinus were noted for 20 patients.

For patients undergoing CT, 8 patients were noted with single sinus involvement, whereas a total of 12 patients had multiple sinus involvement among which 3 patients had two sinuses involved, 6 patients had three sinuses involved, and 3 patients had all four sinuses involved. For patients undergoing MRI examination, 12 patients have single sinus involved, whereas 8 patients had multiple sinuses involved among which 4 patients got two sinuses involved followed by three sinuses involvement in 3 patients and all four sinuses involvement was noted only on 1 patient.

\section{CT findings in paranasal sinus}

This study revealed that 16 patients were having DNS, among which 10 cases had nasal septum deviated toward the left side and 6 cases had nasal septum deviated toward the right side. It was also found that 7 cases out of 20 patients were having bony spur formation. The occurrence of concha bullosa was seen in a total of 5 patients, among which 3 cases were unilateral and 2 cases were bilateral

\section{MRI findings in paranasal sinus}

For the patients undergoing the MRI, the mucosal thickening of maxillary sinus was measured in $\mathrm{mm}$ and it was found that a total of 10 patients had a mucosal thickness of $<5 \mathrm{~mm}, 4$ patients had $5-10 \mathrm{~mm}$ thickness, and 2 patients had a thickness $>10 \mathrm{~mm}$.

The hyperintensity of mucosal thickening is due to water content in the lining; this will determine the type of sinusitis. In the present study, 6 patients had mild, 8 patients had moderate, and 2 patients have severe hyperintensity (Fig. 2).

It was found that 11 patients undergoing MRI had the DNS. Among these, 7 patients had the hypertrophy of inferior turbinate bone (Figs. 3 and 4).

\section{DISCUSSION}

The paranasal sinus is prone to infections due to blockage or narrowing of ostium or osteomeatal complex of paranasal sinus due to different

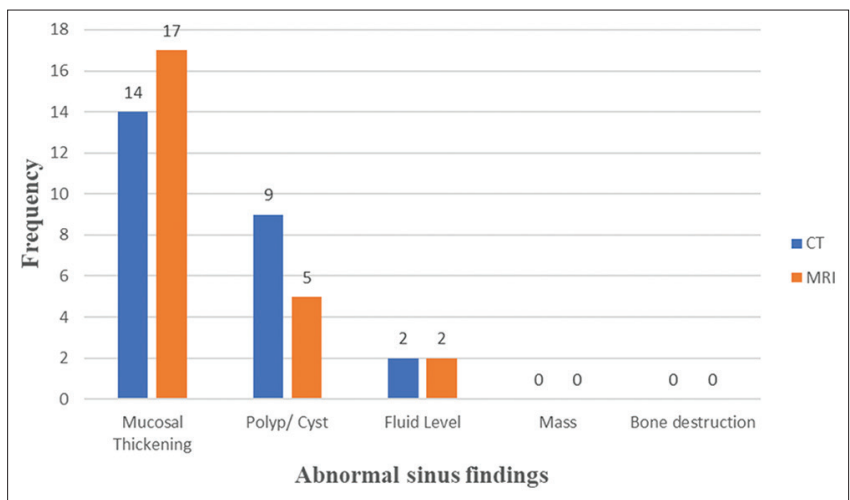

Fig. 1: Distribution of abnormal sinus findings among the computed tomography and magnetic resonance imaging patients expressed as mucosal thickening, polyp/cyst, fluid level, bone destruction, and mass

Table 1: Distribution of pathological condition and pattern of involvement in different sinuses

\begin{tabular}{|c|c|c|c|c|c|c|}
\hline \multirow[t]{2}{*}{ PNS } & \multicolumn{3}{|c|}{ CT findings $(n=20)$} & \multicolumn{3}{|c|}{ MRI findings (n=20) } \\
\hline & f & Unilateral involvement & Bilateral involvement & f & Unilateral involvement & Bilateral involvement \\
\hline Maxillary & 18 & 10 out of 18 & 8 out of 18 & 16 & 9 out of 16 & 7 out of 16 \\
\hline Frontal & 7 & 6 out of 7 & 1 out of 7 & 6 & 5 out of 6 & 1 out of 6 \\
\hline Ethmoid & 10 & 5 out of 10 & 5 out of 10 & 7 & 1 out of 7 & 6 out of 7 \\
\hline Sphenoid & 7 & - & - & 2 & - & - \\
\hline
\end{tabular}

$\mathrm{N}=$ Number of patients, $\mathrm{f}=$ Frequency of sinus involvement; multiple sinus involvement 


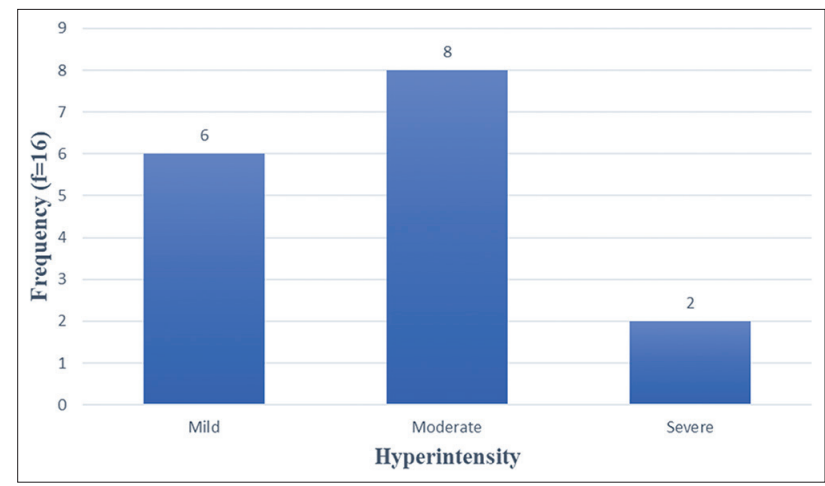

Fig. 2: Distribution showing the different grades of hyperintensity in mucosal lining of maxillary sinus in T2-weighted images

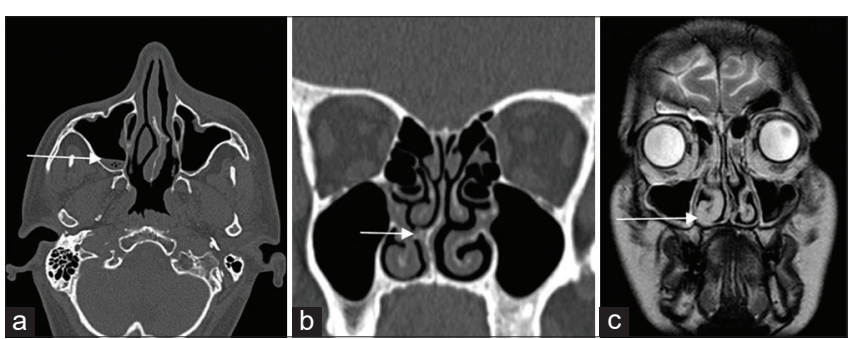

Fig. 3: (a) Axial NCCT image showing air-fluid level at right maxillary sinus; (b) coronal NCCT image showing DNS toward the right side with bone spur formation impregnating on the right inferior turbinate; (c) T2-weighted coronal MRI images shows the right inferior turbinate hypertrophy with DNS toward the left side

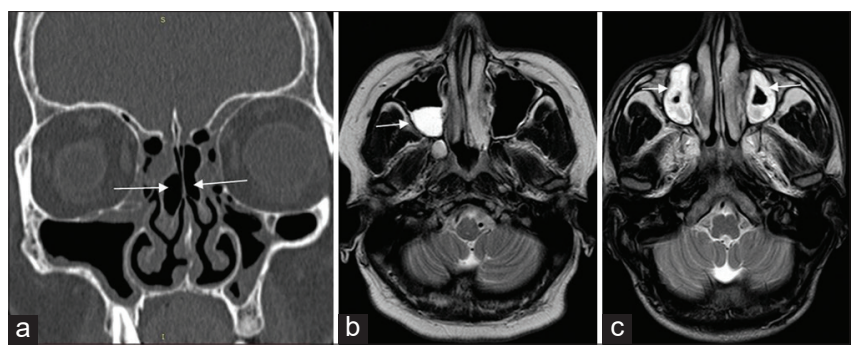

Fig. 4: (a) Coronal NCCT image of PNS in the bone window showing bilateral concha bullosa; (b) T2-weighted axial MRI images show the hyperintense fluid level in the right maxillary sinus; (c) T2-weighted axial MRI images show the hyperintense

thickened sinus mucosa in the bilateral maxillary sinus

etiological factors such as pollutions or anatomical variations occurring within these structures.

In the present study, the most common age group with sinus pathology was 30-40 years, with the highest prevalence among male gender, that is, 23 out of 40 for both patients undergoing CT and MRI scans. This may be due to increased pollution in the Gurugram area due to rapid urbanization and industrialization, and exposure of the male population of this working-age group to a polluted work environment [8].

Sharma et al. conducted a study in which the most common age group affected by sinus pathology was $20-30$ years, male being commonly affected. His results were slightly different from the present study as the geographical location was different from the present study. The most common sinus pathology was polyp (69\%) followed by mucosal thickening. The most often observed anatomical variation was DNS (68\%) followed by concha bullosa. The bone spur formation was not included in the study by literature [9].
In the present study, for patients undergoing CT scans, the most common pathology found was mucosal thickening followed by a polyp and the occurrence air-fluid level which was slightly different from Sharma et al. The most affected paranasal sinus was maxillary sinus followed by ethmoid sinus. This might be because the ostia of the maxillary sinus is located high on the medial wall, thus gravity cannot assist the drainage of mucus and slight changes in the ostia of maxillary sinus renders it liable to infections. Multiple involvements of sinus were reported. The occurrence of DNS was reported in $80 \%$ of cases which was similar to the study conducted by Sharma et al. The appearance of bone spur formation was evident in 7 out of 20 patients and that of concha bullosa in 5 patients.

Maharjan conducted a study "Assessment of PNS in Magnetic Resonance Imaging of brain" among the asymptomatic patients undergoing routine MRI brain study in which the incidental findings were noted. The mucosal thickening was the most common finding, and both mass and fluid levels were least frequent. The highest number of patients were from 15-30 years age group, the male being mostly affected. The mucosal thickening was measured for maxillary sinus and it was found that the maximum patients were having a thickness $<5 \mathrm{~mm}[10]$.

In the present study, among the patients undergoing MRI scans, the most common pathology found was mucosal thickening which was similar to Maharjan, followed by a polyp and the occurrence air-fluid level. Maxillary sinus was found to be the most affected sinus followed by the ethmoid sinus. Since the involvement of mucosal thickening was maximum in the maxillary sinus, the thickness was measured in $\mathrm{mm}$ and it was found that 10 patients were found to have a mucosal thickness $<5 \mathrm{~mm}$ followed by $5-10 \mathrm{~mm}$ and $10 \mathrm{~mm}$ mucosal thickness which was similar to Maharjan. There was a significant amount of mucosal thickening even in patients undergoing the routine MRI brain study without any symptoms of sinus infection. The hyperintensity of the lining of mucosa for maxillary sinus in T2-weighted image was evaluated by reporting radiologists under three categories, 6 cases had mild intensity, 8 cases were having moderate, and 2 cases were found to have severe hyperintensity. The degree of hyperintensity was due to the amount of water content in mucus present within the mucosal lining which differentiates between the acute and chronic sinusitis.

Sahu et al. [11] conducted a study in which he concluded that the severity of DNS and the proportion of inferior turbinate hypertrophy are correlated which was similar to that of present study although the severity was not assessed in present study. The co-occurrence of DNS and inferior turbinate hypertrophy was noted majorly.

Smith et al. performed a study "Prevalence of Concha Bullosa and Nasal Septal Deviation and Their Relationship to Maxillary Sinusitis by Volumetric Tomography" in which the cooccurrence of concha bullosa and nasal septal deviation was found negative, whereas in the present study, it shows positive, this may be because of the small sample size [3]. Although there were certain limitations, the study was able to assess the prevalence and pattern of pathological and anatomical conditions in the Gurugram population. The study clearly shows that the common working-age group was the most affected population as they are exposed to pollution which might be one of the causes to initiate the infections in PNS. This indicates the need for a cross-sectional study that can correlate the relationship between pollution and the prevalence of pathological conditions in PNS under imaging modalities.

\section{Limitation of the study}

Since the incidental findings in the PNS among the routine MRI cases were to be included in the study, the sample size was relatively small. There might be Significant potential bias in the assessment of hyperintensity due to different imaging protocols in MRI. Lack of 
correlation between the clinical symptoms and findings was one of the most evident limitations of the study.

\section{CONCLUSION}

From the present study, it can be concluded that mucosal thickening was commonly evident pathology with maximum occurrence seen in the maxillary sinus. More than 1 anatomical variation and pathology were evident in most of the patients. The working-age population was the most affected one. MRI was sensitive in assessing the early and minute changes in sinus mucosal abnormalities as the hyperintensity of mucosal lining changes if any infection is present.

\section{ACKNOWLEDGMENT}

We are thankful to the Department of Radiodiagnosis and Imaging at Shree Guru Gobind Tricentenary University-Hospital, Gurugram, for their approval and permission in collecting the data and carrying out this study smoothly.

\section{AUTHORS' CONTRIBUTIONS}

All the authors contributed to the preparation of the final manuscript.

\section{CONFLICTS OF INTEREST}

There are no conflicts of interest regarding the publication of this article.

\section{AUTHORS' FUNDING}

Authors did not receive any funding for this research work.

\section{REFERENCES}

1. Verma J, Rathaur SK, Mishra S, Mishra AK. The role of diagnostic imaging in evaluation of nasal and paranasal sinus pathologies. Int J Otorhinolaryngol Head Neck Surg 2016;2:140-6.

2. Rai GS, Rai T, Jain L, Vyas MM, Roshan R. Evaluation of CT and MRI findings among patients presented with chief complaint of headache in central India. J Clin Diagn Res 2016;10:TC21-5.

3. Smith KD, Edwards PC, Saini TS, Norton NS. The prevalence of concha bullosa and nasal septal deviation and their relationship to maxillary sinusitis by volumetric tomography. Int J Dent 2010;2010:404982.

4. Mays S, Mavrogordato M, Lambert J, Sofaer J. The prevalence and health implications of concha bullosa in a population from mediaeval England. Int J Osteoarchaeol 2014;24:614-22.

5. Hussein AO, Ahmed BH, Omer MA, Manafal MF, Elhaj AB. Assessment of clinical, X-ray and CT in diagnosis of paranasal sinus diseases. Int J Sci Res 2014;3:7-11.

6. Pillai S, Bhandarkar AM, Nair SS, Jha AT, Ramaswamy B, Al-Abri R. A radiological profile of fungal sinusitis. Asian J Pharm Clin Res 2017;10:331-4.

7. Lloyd GA, Lund VJ, Phelps PD, Howard DJ. Magnetic resonance imaging in the evaluation of nose and paranasal sinus disease. $\mathrm{Br} \mathrm{J}$ Radiol 1987;60:957-68.

8. Rizwan SA, Nongkynrih B, Gupta SK. Air pollution in Delhi: Its magnitude and effects on health. Indian J Community Med 2013;38:4-8.

9. Sharma BN, Panta OB, Lohani B, Khanal U. Computed tomography in the evaluation of pathological lesions of paranasal sinuses. J Nepal Health Res Counc 2015;13:116-20.

10. Maharjan S. Assessment of paranasal sinuses in magnetic resonance imaging of brain. Bangabandhu Sheikh Mujib Med Univ J 2016;9:104-6.

11. Sahu N, Mohapatra SS, Rath SN, Padhy RN. Computed tomographic evaluation of inferior turbinate hypertrophy in adult Indo-Dravidian patients with deviated nasal septum. Asian J Pharm Clin Res 2017;10:342-4. 